\title{
Effects of sympathetic modulation in metabolic disease
}

\author{
Revathy Carnagarin ${ }^{1}$, Gavin W. Lambert ${ }^{2,3}$, Marcio G. Kiuchi ${ }^{1}$, Janis M. Nolde ${ }^{1}$, Nina \\ Eikelis $^{2,3}$, Elisabeth A. Lambert ${ }^{2,3}$, Markus P. Schlaich ${ }^{1,4,5}$.
}

${ }^{1}$ Dobney Hypertension Centre, School of Medicine - Royal Perth Hospital Unit / Medical Research Foundation, University of Western Australia; ${ }^{2}$ Iverson Health Innovation Research Institute and ${ }^{3}$ School of Health Sciences, Swinburne University of Technology, Hawthorn, Australia, ${ }^{4}$ Departments of Cardiology and Nephrology, Royal Perth Hospital, Perth, Australia; ${ }^{5}$ Neurovascular Hypertension \& Kidney Disease Laboratory, Baker Heart and Diabetes Institute, Melbourne, Australia.

Total word count: 5836 words (includes references)

Key words: $\quad$ sympathetic nervous system, hypertension, renal denervation, metabolic diseases, glucose metabolism, norepinephrine

Correspondence to:

Winthrop Professor Markus Schlaich

Dobney Chair in Clinical Research

School of Medicine - Royal Perth Hospital Unit

The University of Western Australia

Level 3, MRF Building, Rear 50 Murray St,

Perth WA 6000; Australia

Phone: +61 892240382 Fax: +61 892240374

e-mail: markus.schlaich@uwa.edu.au

This is the peer reviewed version of the following article: Carnagarin, R., Lambert, G.W., Kiuchi, M.G., Nolde, J.M., Matthews, V.B., Eikelis, N., Lambert, E.A. and Schlaich, M.P. (2019), Effects of sympathetic modulation in metabolic disease. Ann. N.Y. Acad. Sci., 1454: 80-89, which has been published in final form at https://doi.org/10.1111/nyas.14217. This article may be used for non-commercial purposes in accordance with Wiley Terms and Conditions for Use of SelfArchived Versions. 


\begin{abstract}
Sympathetic overdrive contributes to the derangement of glucose metabolism evident in clinical conditions such as obesity, metabolic syndrome, type 2 diabetes, obstructive sleep apnea and others. Targeting the sympathetic nervous system directly therefore appears as an attractive therapeutic approach to restore impaired glucose metabolism. Indeed, lifestyle interventions including healthier diets and exercise have been shown to exert their beneficial effects at least in part by reducing sympathetic nervous system activity. Pharmacologic inhibition of exaggerated central sympathetic outflow has also been demonstrated to beneficially impact on body weight, glucose and lipid metabolism. More recently, catheterbased renal denervation, an intervention applied predominantly to lower elevated blood pressure in patients with resistant hypertension, revealed salutary effects on glucose metabolism. Here, we review the mechanisms that contribute to the beneficial effects of targeting the sympathetic nervous system directly and discuss how these approaches may best be embedded in routine clinical practice.
\end{abstract}




\section{Introduction}

The sympathetic nervous system (SNS), once thought to predominantly cater for the extreme flight and fight responses, in fact plays an integrative regulatory role to sustain cardiovascular and metabolic homeostasis. Central sympathetic overdrive is the hallmark of several important clinical conditions including hypertension (HTN) and cardiovascular, renal and metabolic disorders. Under normal circumstances, sympathetic outflow to the heart increases cardiac output and facilitates neurally mediated vasoconstriction of the peripheral vasculature to maintain blood pressure (BP) levels. Renal sympathetic outflow impacts renal hemodynamics, increases renin release thereby activating the renin angiotensin system (RAS) cascade, and causes sodium and water retention to regulate $\mathrm{BP}^{1}$. Of note hypertension often coexists with obesity and other metabolic disorders ${ }^{2}$. In addition to well-described effects on cardiometabolic conditions, SNS activation differentially regulates immune-mediated pathways to sustain the inflammation $^{3}$ implicated in the derangement of metabolic homeostasis and is evident in patients with diabetes, patients with obesity, and patients with the metabolic syndrome ${ }^{4}$. Sympathetically -induced chronic inflammation ${ }^{5}$ seems to contribute substantially to both acute and long-term BP elevations and its adverse consequences ${ }^{6,7}$ [Fig 1].

Targeting sympathetic activation therapeutically appears as a logical approach not only in the management of cardiovascular disorders such as hypertension and its complications but likely also in metabolic conditions associated with sympathetic activation. Aside from lifestyle interventions, the strategies used to achieve SNS inhibition are pharmacotherapy and devicebased approaches. Though the pharmacological approaches are safe, management of resistant hypertension becomes increasingly difficult with metabolic co-morbidities partially due to inadequate targeting of the $\mathrm{SNS}^{8}$. Hence sympathetic ablation using device-based therapeutic strategies such as renal denervation and carotid body ablation gained interest as hypertension in many patients remained poorly controlled with pharmacotherapy alone. Whilst carotid body 
ablation is still at its nascent stages, accumulating evidence suggests that renal denervation is a viable option to target sympathetic activation in the management of treatment-resistant hypertension and other clinical conditions associated with increased sympathetic drive ${ }^{9}$ with the benefit of salutary effects on glucose metabolism.

\section{Renal Sympathetic Denervation for targeting sympathetic overdrive.}

Renal artery denervation (RDN) though reported as early as $1924^{10}$, has re-emerged as a promising treatment for hypertension recently owing to its multitude of cardiovascular and metabolic benefits including improved blood pressure control, regression of left ventricular hypertrophy and diastolic dysfunction, as well as improved insulin sensitivity and lipid profile 11-14. However, the clinical studies that assessed the overall effect of RDN as a possible therapeutic option has shown diverse results ${ }^{12,15-17}$. The first proof-of-concept trial, SYMPLICITY HTN-1 that evaluated RDN, demonstrated that catheter-based denervation led to a substantial BP reduction without any serious adverse events, ${ }^{11}$ both at 6 months and subsequent follow ups compared with standard antihypertensive pharmacotherapy ${ }^{18,19}$. Following this, SIMPLICITY HTN-2 trial showed similar significant blood pressure reduction in the RDN group compared to the control subjects ${ }^{12}$. However, both these were limited in that not blinded and did not include a sham-controlled design. Other limitations included incomplete standardisation of blood pressure measurement and absence of assessment of adherence to antihypertensive medication. The SYMPLICITY HTN-3 trial, designed as a prospective, sham controlled study thereby addressing the shortcomings of the initial RDN trials failed to demonstrate a more pronounced BP lowering effect of RDN compared to a shamprocedure ${ }^{19,20}$. 
The outcome of the SYMPLICITY HTN-3 trial tossed RDN into hibernation despite criticism relating to study conduct, incomplete denervation and lack of circumferential, four quadrant sympathetic fibre interruption. The SYMPLICITY HTN-4 clinical trial that was planned to follow SYMPLICITY HTN-3 to investigate patients with moderate uncontrolled HTN was halted as SYMPLICITY HTN-3 failed to meet its primary efficacy endpoint. However, the DENER-HTN trial, designed to address the major concern of lack of antihypertensive standardization and adherence in the SYMPLICITY HTN-3 trial clearly demonstrated the supremacy of RDN when delivered with a standardized stepped-care antihypertensive treatment approach over the same standardized stepped-care antihypertensive treatment alone 21. Clearly, SYMPLICITY HTN-3 and DENERHTN trials identified the need to characterize patient populations that may benefit most from $\mathrm{RDN}^{22}$. In support of this view, the study by Kario et al indicated that patients with concomitant obstructive sleep apnea seemed to respond more favourably to $\mathrm{RDN}^{23}$.

Importantly, trial designs were adjusted to overcome issues identified in previous studies and three rigorously executed sham controlled studies with similar protocol design were recently published (SPYRAL HTN-OFF MED, SPYRAL HTN-ON MED, and RADIANCE-HTN SOLO ${ }^{24-26}$ ) and all consistently demonstrated a significant BP effect compared to sham control [Fig 2]. However, the magnitude of BP reduction achieved was smaller in comparison to the uncontrolled, unblinded RDN studies and was similar to changes expected from drug therapy modification $^{19,22,27}$.

In addition, these studies excluded patients with isolated systolic hypertension who form around $70 \%$ of the elderly population, thereby data from these studies are not applicable to this important group. Hence, future trials are still warranted to reconcile the true efficacy of RDN in the treatment of hypertension and various other cardiac and metabolic conditions. Nevertheless, RDN is again considered a valuable alternative to lower BP in clinical practice. 
Indeed, results from these studies prompted a meeting of the US Food and Drug Administration Circulatory System Devices Panel in December 2018, to discuss recommendations regarding clinical evaluation of anti-hypertensive devices ${ }^{28}$. It is hence worthwhile to revisit the mechanisms underlying the effects of renal denervation, and its potential in the management of cardio metabolic disorders.

\section{Mechanisms of RDN-induced clinical benefits}

The innervation of the kidneys includes efferent sympathetic fibres originating from central nervous system nuclei traversing to the kidney as well as afferent sensory fibres from the kidneys projecting to integrating nuclei in the brainstem [Fig 3]. Increased central sympathetic outflow directed towards the kidneys reduces renal blood flow, stimulates renin release, and enhances the tubular reabsorption of sodium and water ${ }^{29}$. Studies in rat models reported that RDN decreases blood pressure independent of renin release or sodium-water balance and did not impact the salt sensitivity of arterial pressure ${ }^{30-33}$. In addition, the afferent sympathetic signalling also contributes to the off target antiarrhythmic effect and other metabolic and longterm effects of RDN. Animal studies have shown that bilateral RDN led to substantial neuronal remodelling in the brain stem and stellate ganglia following RDN procedure ${ }^{34-37}$. The renal efferent sympathetic nerves arising from the second sympathetic ganglion, form a network within the renal artery adventitia ${ }^{38}$ which is regulated by the rostral ventrolateral medulla (RVLM), the destruction of which substantially reduces blood pressure ${ }^{39}$. Accordingly, the RDN procedure was introduced to disrupt the sympathetic cross-talk from the kidneys to the brain and vice versa as well as between the two kidneys ${ }^{40}$. Sympathetic ablation by RDN, evident from the reduction in markers of sympathetic activity such as MSNA and noradrenaline spillover ${ }^{41}$, reduces vasoconstriction, improves metabolism and cardiac performance, with favourable renal effects ${ }^{42}$. Moreover, RDN blocks adverse renal stimuli 
(i.e. renal ischemia, electrolyte imbalance, oliguria, high adenosine levels) from being projected to the relevant brain regions in turn attenuating central sympathetic outflow to other organs.

In addition, RDN ameliorates SNS-dependent modulation of immune pathways that causes metabolic perturbations such as insulin resistance and hyperinsulinemia and vice versa. Sympathetic activation triggers inflammation of the skeletal muscle vasculature, brain, and kidneys and contributes to chronic elevation in $\mathrm{BP}^{43-45}$ along with metabolic disturbances. In clinical conditions associated with high sympathetic drive, a chronic systemic low-grade inflammatory environment is established and sustained by activated immune cells and increased cytokines in the circulation ${ }^{46-50}$. Distinct clinical states such as obesity, metabolic syndrome and type 2 diabetes as well as obesity related hypertension are characterised by immune activation and increased circulating inflammatory cytokines and interleukins exerting metabolic stress, perturbing systemic homeostasis ${ }^{49-54}$. Bilateral chemical ablation of renal sympathetic nerves in mice inhibited immune activation, renal inflammation and attenuated Ang II-induced hypertension ${ }^{55}$. Similarly, RDN attenuated hypertension and renal inflammation in the rat deoxycorticosterone acetate-salt model of hypertension, which was driven by renal inflammation-induced augmented afferent sympathetic trafficking ${ }^{56}$. In addition, catheter-based renal denervation induced reduction in SNS activity not only lowered BP but also reduced monocyte activation and inflammatory cytokines in patients with arterial hypertension ${ }^{57}$.

\section{The metabolic effects of RDN}

Heightened sympathetic activation induces insulin resistance ${ }^{58}$ and obesity ${ }^{59}$ and contributes to the development of diabetes mellitus ${ }^{60}$ and metabolic syndrome ${ }^{61}$ with the resultant hyperinsulinemia causing further sympatho-excitation ${ }^{62,63}$. Around $50 \%$ of patients with 
essential hypertension are insulin resistant, ${ }^{64}$ and hypertension often coexists with obesity ${ }^{65,66}$. Increased adiposity, insulin resistance and hyperinsulinemia have been directly related to enhanced muscle sympathetic nerve activity (MSNA) activity ${ }^{67,68}$. The vascular resistance induced by sympathetic activation causes a shift of blood flow from striated muscle to viscera, which is less insulin sensitive than striated muscle. In addition, the prevalence of hypertension is associated with impaired postprandial insulinemia ${ }^{69}$. Sympathetic inhibition by dietary measures predicts the weight loss in metabolic syndrome ${ }^{70,71}$. These findings form the pathophysiological rationale for the introduction of RDN as potential strategy for amelioration of perturbed metabolic homeostasis.

Inhibition of central sympathetic outflow with the centrally acting imidazline receptor agonist moxonidine is associated with improvement of glucose metabolism by increasing the skeletal blood flow, decreasing glucagon secretion that accompanies reduced glycogenolysis and gluconeogenesis ${ }^{72}$. However, the use of centrally acting sympatholytic agents can be limited by side effects, leading to relatively high non-adherence rates. ${ }^{73}$ In this context RDN is a potential option to ameliorate glucose metabolism ${ }^{74}$. RDN reduces norepinephrine release, attenuates $\alpha$-adrenergic tone, and reduces RAS activation with salutary consequences on striated muscle blood flow ${ }^{75}$. This improvement in peripheral blood flow in turn facilitates insulin delivery to the skeletal muscle enhancing glucose uptake and utilisation ${ }^{76}$. Sympathetic overactivity renders hypertensive patients more susceptible to weight gain associated with reduced responsiveness of adrenoceptors ${ }^{77}$. Increased SNS activity promotes insulin resistance and central adiposity, which in turn has been associated with higher rates of sleep apnea ${ }^{78}$ and further exacerbation of impaired glucose metabolism. In obese patients with resistant hypertension and concomitant obstructive sleep apnea, attenuation of sympathetic overdrive induced significant weight 
loss ${ }^{79}$, ameliorated $\mathrm{BP}$ and improved metabolic parameters ${ }^{76,78}$. Taken together, RDN could provide a sustainable therapeutic option in obesity related hypertension, particularly in those with concomitant sleep apnea, to improve BP and possibly metabolic homeostasis. Indeed, clinical data suggest that RDN is a potentially valuable intervention in metabolic diseases associated with BP elevation with a likely reduction in cardiovascular risk. RDN besides reducing $\mathrm{BP}$, improved glycosylated haemoglobin levels and attenuated sleep apnea severity with improvement in indices of insulin action and metabolic parameters in patients with resistant hypertension ${ }^{72,79}$. The EnligHTN I trial ${ }^{80}$ showed that RDN could potentially benefit patients with heart failure as there was a significant reduction of average heart rate $^{81}$. In addition, RDN was associated with reduction in left ventricular mass, improvement of atrial and ventricular arrhythmias ${ }^{82}$ and diastolic parameters as well ${ }^{83}$. Moreover, RDN improved indices of renal resistance and ameliorated the severity of macroalbuminuria and microalbuminuria ${ }^{84}$. Interestingly, in hypertensive patients with diabetes mellitus, RDN improved glucose metabolism and insulin sensitivity ${ }^{72,78}$. Furthermore, the benefits of RDN has been extended to combined metabolic and endocrine disorders. In polycystic ovary syndrome, RDN improved blood pressure and fasting glucose with parallel improvement in insulin sensitivity along with a reduction in glomerular hyperfiltration and urinary albumin excretion ${ }^{85}$. RDN also slows the progression of renal injury in diabetes which had already been proven in an animal $\operatorname{model}^{86}$.

However, the DREAMS-Study ${ }^{87}$, investigating the effects of RDN on blood pressure and insulin sensitivity in patients with metabolic syndrome neither showed a change in fasting glucose, insulin sensitivity nor improved sympathetic activity ${ }^{88}$. The DREAMS-Study included nearly drug-naïve patients with higher sympathetic activity compared to other studies $^{89,90}$ and would have been expected to demonstrate at least a reduction in 
sympathetic activity ${ }^{91}$. Whether insufficient denervation may have contributed to this finding is uncertain but remains a possibility. The study lacked a control group and the small size of the cohort makes it difficult to draw any firm conclusions. Further evaluation in the form of rigorously designed clinical trials are mandatory and ongoing to confirm the previously reported beneficial effects of RDN in diabetes mellitus.

\section{Limitations and potential complications of renal denervation}

It is now exactly 10 years ago that the results from Symplicity HTN-1, the first safety and proof-of-concept study applying RDN in patients with resistant hypertension was published ${ }^{11}$. However, longer term follow up data is thus far only available for up to three years from Symplicity HTN-1 ${ }^{18}$, Symplicity HTN-2 ${ }^{94}$, and from the Global Symplicity Registry ${ }^{95}$. This is mainly due to the fact that follow up was only planned for three years following the procedure. Although safety of the procedure was demonstrated in all these studies, longer term follow up data together with reporting of cardiovascular and renal endpoints would be useful. The Global Symplicity Registry is collecting these data and will help to close this gap in the future. A very recent meta-analysis reviewing the efficacy and safety of renal denervation within the 6 trials that had a sham-control arm ${ }^{96}$, reported that no major peri-procedural adverse events were reported in either group in 5 of the 6 trials and that the SYMPLICITY HTN-3 trial reported major adverse events in $1.4 \%$ of the RDN group and $0.6 \%$ of the sham controlled group. They concluded that patients treated with RDN experienced significantly greater reduction in 24-h ambulatory systolic blood pressure and that adverse events associated with the RDN procedures were rare ${ }^{96}$. While these findings are reassuring long term safety and efficacy will have to be monitored in ongoing studies. 


\section{Effects of other interventional sympatholytic approaches on glucose metabolism}

Baroreflex activation therapy (BAT) is another relevant intervention targeting sympathetic pathways. Indeed, the procedure has been demonstrated to significantly reduce arterial blood pressure (BP) by inhibition of the sympathetic nervous system. The effect of this approach on the metabolic state has been tested in patients with resistant hypertension. Wallbach et al. ${ }^{97}$ prospectively assessed the effects of BAT in a cohort of 30 patients with resistant hypertension on blood pressure, BMI, weight, fasting glucose, insulin, C-peptide, HbA1c, HOMA-IR, HOMA-b, ISQuickI, and glucose levels during oral glucose tolerance test, both at baseline and 6 months after the procedure. While fasting glucose levels were significantly reduced at 6 months follow up, all other parameters remained essentially unchanged. The authors concluded that BAT is effective in reducing BP but may only have minimal effects on the metabolic state at 6 months. In line with these findings, a study in obese hypertensive dogs exposed to BAT revealed sustained reductions in $\mathrm{BP}$, heart rate and plasma norepinephrine concentrations, but no changes in plasma glucose or insulin concentrations ${ }^{98}$. Together, these findings may indicate that potential beneficial effects of interventions to lower sympathetic activity may have disparate effects on glucose metabolism.

\section{Future Perspectives and Conclusions}

The exact mechanisms by which RDN impacts on insulin metabolism and glucose homeostasis remain to be fully understood. They are likely to involve a concerted interplay of complex mechanisms such as modulation of afferent signaling to reduce central sympathetic outflow, interference with the RAS cascade, a decrease in alpha adrenergic vascular tone dilating skeletal muscle vasculature, increased insulin and non-esterified fatty acid sensitivity, and improved glucose uptake and transport to skeletal muscle ${ }^{91-93}$. Recent studies applying RDN in patients with diabetes have several limitations including small sample size, lack of adequate control groups, short follow up and others. Beneficial 
effect of the RDN induced BP reduction per se which could account for the improvements in glucose metabolism cannot be excluded. Future studies in the metabolic area are required to address these limitations and to confirm the previous results and enhance the understanding of the potential beneficial impact of RDN on metabolism in patients with metabolic syndrome and diabetes. The results of ongoing RDN clinical trials in diabetes (NCT 02081989) and metabolic syndrome (NCT 01911078) performed to determine the effects of sympathetic ablation by RDN on glucose metabolism and insulin sensitivity, will further define the role and applicability of RDN in metabolic disorders.

\section{Disclosures}

The laboratories of GL and MS have recently received research funding from Medtronic, Abbott, and Servier Australia. GL has received honoraria or travel support from Medtronic, Pfizer, and Wyeth. MS serves on scientific advisory boards for Abbott, BI, Novartis, and Medtronic and has received honoraria and travel support from Abbott, BI, Servier, Novartis, and Medtronic. MS is supported by an NHMRC Senior Research Fellowship. 


\section{References}

1. DiBona, G.F. \& U.C. Kopp. 1997. Neural control of renal function. Physiol. Rev. 77: 75-197.

2. Carnagarin, R., C. Gregory, O. Azzam, et al. 2017. The Role of Sympatho-Inhibition in Combination Treatment of Obesity-Related Hypertension. Curr. Hypertens. Rep. 19: 99.

3. Carnagarin, R., V. Matthews, M.T.K .Zaldivia, et al. 2018. The bidirectional interaction between the sympathetic nervous system and immune mechanisms in the pathogenesis of hypertension. Br. J. Pharmacol. 10.1111/bph.14481

4. Lambert, G.W., N.E. Straznicky, E.A. Lambert, et al. 2010. Sympathetic nervous activation in obesity and the metabolic syndrome-causes, consequences and therapeutic implications. Pharmacol Ther 126: 159-172.

5. Janse van Rensburg, D.C., J.A. Ker, C.C. Grant \& L. Fletcher. 2012. Autonomic impairment in rheumatoid arthritis. Int. J. Rheum. Dis. 15:419-426.

6. Esler, M.. 2000. The sympathetic system and hypertension. Am. J. Hypertens. 13: 99s$105 \mathrm{~s}$.

7. Schlaich, M.P., P.A. Sobotka, H. Krum, E. Lambert \& M.D. Esler. 2009. Renal sympathetic-nerve ablation for uncontrolled hypertension. N. Engl. J. Med. 361:932934.

8. Fisher J.P. \& P.J. Fadel. 2010. Therapeutic strategies for targeting excessive central sympathetic activation in human hypertension. Exp. Physiol. 95:572-580. https://doi.org/10.1113/expphysiol.2009.047332

9. Schlaich, M.P. 2017. Renal Sympathetic Denervation: A Viable Option for Treating Resistant Hypertension. Am. J. Hypertens. 30: 847-856.

10. Smithwick R.H.. 1949. An evaluation of the surgical treatment of hypertension. Bull. N. Y. Acad. Med. 25: 698-716. 
11. Krum H., M. Schlaich, R. Whitbourn, P.A. Sobotka, et al. 2009. Catheter-based renal sympathetic denervation for resistant hypertension: a multiticentre safety and proof-ofprinciple cohort study. Lancet 373: 1275-1281.

12. Esler M.D., H. Krum, P.A. Sobotka, M.P. Schlaich, et al. 2010. Renal sympathetic denervation in patients with treatment-resistant hypertension (The Symplicity HTN-2 Trial): a randomised controlled trial. Lancet 376: 1903-1909.

13. Symplicity HTN-1 Investigators. 2011. Catheter-based renal sympathetic denervation for resistant hypertension: durability of blood pressure reduction out to 24 months. Hypertension 57: 911-917.

14. Maraj S., R. Patel, R. Oliveros, et al. 2012. Potential Cardiometabolic Benefits of Renal Artery Denervation in Diabetics. J. Diabetes. Metab. S3:007. doi:10.4172/2155-6156.S3007.

15. Ram V.S. \& A.S Kumar. 2014. Renal denervation therapy for resistant hypertension: a clinical update, J. Hum. Hypertens 12: 699-704.

16. Bhatt D.L., D.E. Kandzari, W.W. O'Neill, et al. 2014. A controlled trial of renal denervation for resistant hypertension. N. Engl. J. Med. 370: 1393-1401.

17. Desch S., T. Okon, D. Heinemann, K. Kulle, et al. 2015. Randomized sham-controlled trial of renal sympathetic denervation in mild resistant hypertension. Hypertension 65 : $1202-1208$

18. Krum H., M.P Schlaich, P.A. Sobotka, M. Böhm, et al. 2014. Percutaneous renal denervation in patients with treatment-resistant hypertension: final 3-year report of the Symplicity HTN-1 study. Lancet 383:622-629. https://doi.org/10.1016/S0140$\underline{6736(13) 62192-3 .}$ 
19. Bakris G.L., R.R. Townsend, M. Liu, S.A. Cohen, et al. 2014. Impact of renal denervation on 24-hour ambulatory blood pressure: results from SYMPLICITY HTN-3. J. Am. Coll. Cardiol. 64:1071-1078. https://doi.org/10.1016/j.jacc.2014.05.012

20. Bakris G.L., R.R. Townsend, J.M. Flack, S. Brar, et al. 2015. 12-month blood pressure results of catheter-based renal artery denervation for resistant hypertension: the SYMPLICITY HTN-3 trial. J.A.C.C. 65:1314-1321. https://doi.org/10.1016/j.jacc.2015.01.037.

21. Azizi M., M. Sapoval, P. Gosse et al. 2015. Optimum and stepped care standardised antihypertensive treatment with or without renal denervation for resistant hypertension (DENERHTN): a multicentre, open-label, randomised controlled trial. Lancet 385:19571965. https://doi.org/10.1016/S0140-6736(14)61942-5.

22. Azizi M., H. Pereira, I. Hamdidouche et al. 2016. Adherence to Antihypertensive Treatment and the Blood Pressure Lowering Effects of Renal Denervation in the Renal Denervation for Hypertension (DENERHTN) Trial. Circulation 134: 847-857. https://doi.org/10.1161/circulationaha.116.022922.

23. Kario K., D.L. Bhatt, D.E. Kandzari et al. 2016. Impact of renal denervation on patients with obstructive sleep apnea and resistant hypertension-insights from the SYMPLICITY HTN-3 trial. Circ. J. 80:1404-1412. https://doi.org/10. 1253/circj.CJ-160035 .

24. Townsend R.R., F. Mahfoud, D.E Kandzari, et al. 2017. SPYRAL HTN OFF MED Trial Investigators*. Catheter-based renal denervation in patients with uncontrolled hypertension in the absence of antihypertensive medications (SPYRAL HTN-OFF MED): a randomised, sham-controlled, proof-of-concept trial. Lancet 390:2160-2170. doi: 10.1016/S0140-6736(17)32281-X 
25. Kandzari D.E., M. Böhm ,F. Mahfoud et al. 2018. SPYRAL HTN-ON MED Trial Investigators. Effect of renal denervation on blood pressure in the presence of antihypertensive drugs: 6-month efficacy and safety results from the SPYRAL HTN-ON MED proof-of-concept randomised trial. Lancet 391:2346-2355. doi: 10.1016/S01406736(18)30951-6

26. Azizi M., R.E. Schmieder, F. Mahfoud et al. 2018. RADIANCE-HTN Investigators. Endovascular ultrasound renal denervation to treat hypertension (RADIANCE-HTN SOLO): a multicentre, international, single-blind, randomised, sham-controlled trial. Lancet 391:2335 - 2345. doi: 10.1016/S0140-6736(18)31082-1.

27. Papademetriou V., C.P. Tsioufis, A. Sinhal, et al. 2014. Catheter-based renal denervation for resistant hypertension: 12-month results of the EnligHTN I firstin-human study using a multielectrode ablation system. Hypertension 64:565-572. doi: 10.1161/HYPERTENSIONAHA.114.03605

28. Food and Drug Administration. FDA Executive Summary. Circulatory System Devices Panel Meeting, December 5, 2018. General Issues Panel Clinical Evaluation of AntiHypertensive Devices. https://www.fda. gov/downloads/AdvisoryCommittees/CommitteesMeetingMaterials/ MedicalDevices/MedicalDevicesAdvisoryCommittee/CirculatorySystem DevicesPanel/UCM626389.pdf

29. Yim H.E. \& K.H. Yoo. 2008. Renin-angiotensin system-considerations for hypertension and kidney. Electrolyte Blood Press. 6:4250. https://doi.org/10.5049/EBP.2008.6.1.42.

30. DiBona G.F. \& M. Esler. 2010. Translational medicine: the antihypertensive effect of renal denervation. Am. J. Physiol. 298: R245-R253. 
31. Foss J.D., G.D. Fink \& J.W. Osborn. 2013. Reversal of Genetic Salt-Sensitive Hypertension by Targeted Sympathetic Ablation. Hypertension 61:806-811.

32. Jacob F., P. Ariza \& J.W. Osborn. 2003. Renal denervation chronically lowers arterial pressure independent of dietary sodium intake in normal rats. Am. J. Physiol-Heart C 284: H2302-H2310.

33. Jacob F., B.G. LaBine, P. Ariza et al. 2005. Renal denervation causes chronic hypotension in rats: Role of beta(1)-adrenoceptor activity. Clin. Exp. Pharmacol. P. $32: 255-262$.

34. Tsai W.C., Y.H. Chan, K. Chinda et al. 2017. Effects of renal sympathetic denervation on the stellate ganglion and brain stem in dogs. Heart Rhythm 14:255-262.

35. Sripairojthikoon W. \& J.M Wyss. 1987. Cells of origin of the sympathetic renal innervation in rat. Am. J. Physiol.252:F957-F963.

36. Gattone V.H., C.F. Marfurt \& S. Dallie. 1986. Extrinsic innervation of the rat kidney: a retrograde tracing study. Am. J. Physiol. 250:F189-F196.

37. Ferguson M., G.B. Ryan \& C. Bell.1986. Localization of sympathetic and sensory neurons innervating the rat kidney. J. Auton. Nerv. Syst. 16:279-688.

38. Nishi E.E., C.T. Bergamaschi \& R.R. Campos. 2015. The crosstalk between the kidney and the central nervous system: the role of renal nerves in blood pressure regulation. Exp. Physiol. 100:479-484. https://doi.org/10.1113/expphysiol.2014.079889.

39. Guertzenstein P.G \& A. Silver.1974. Fall in blood pressure produced from discrete regions of the ventral surface of the medulla by glycine and lesions. J Physiol 242:489503.

40. Sobotka P., F. Mahfoud, M. Schlaich et al. 2011. Sympatho-renal axis in chronic disease. Clin Res Cardiol 100: 1049-1057. 
41. Schlaich M., P. Sobotka, H. Krum \& E. Lambert. 2009. Renal sympathetic nerve ablation for uncontrolled hypertension. N. Engl. J. Med. 361: 932-934.

42. De Miguel C., N.P. Rudemiller, J.M. Abais \& D.L. Mattson. 2015. Inflammation and hypertension: new understandings and potential therapeutic targets. Curr. Hypertens. Rep.17:507.

43. McMaster W.G., Kirabo A., Madhur M.S. \& Harrison D.G. 2015. Inflammation, immunity, and hypertensive end-organ damage. Circ. Res.116:1022-33.

44. Zubcevic J., M.M. Santisteban, T. Pitts et al.2014. Functional neural-bone marrow pathways: implications in hypertension and cardiovascular disease. Hypertension 63:e129-139.

45. Heidt T., H.B. Sager, G. Courties et al. 2014. Chronic variable stress activates hematopoietic stem cells. Nat Med 20: 754-758.

46. Harrison D. 2014. The immune system in hypertension, Transactions of the American Clinical and Climatological Association 125: 130-138.

47. Dörffel Y., C. Lätsch, B. Stuhlmüller et al. 1994. Preactivated peripheral blood monocytes in patients with essential hypertension. Hypertension 34: 113-117.

48. Wenzel P., M. Knorr, S. Kossmann et al. 2011. Lysozyme M-positive monocytes mediate angiotensin II-induced arterial hypertension and vascular dysfunction. Circulation 124: $1370-1381$.

49. Shoelson S., J. Lee \& A. B. Goldfine. 2006. Inflammation and insulin resistance. J. Clin. Invest. 116: 1793-1801.

50. Chen L., Chen R., Wang H. \& Liang F. 2015. Mechanisms linking inflammation to insulin resistance. Int. J. Endocrinol. 2015: ID 508409.

51. Maestroni G.J. 2000. Dendritic cell migration controlled by $\alpha 1$ badrenergic receptors. J. Immunol. 165: 6743-6747. 
52. Heijnen B.F., H. Van Essen, C.G. Schalkwijk, B.J. Janssen \& H.A. Struijker-Boudier. 2014. Renal inflammatory markers during the onset of hypertension in spontaneously hypertensive rats. Hypertens. Res. 37:100-109.

53. Perez D.M., R.S. Papay \& T. Shi. 2009. $\alpha 1$-Adrenergic receptor stimulates interleukin-6 expression and secretion through both mRNA stability and transcriptional regulation: involvement of p38 mitogen activated protein kinase and nuclear factor- $\kappa \mathrm{B}$. Mol. Pharmacol. 76: 144-152.

54. Grisanti L.A., A.P. Woster, J. Dahlman et al. 2011. $\alpha 1$-Adrenergic receptors positively regulate toll-like receptor cytokine production from human monocytes and macrophages. J. Pharmacol. Exp. Ther. 338: 648-657.

55. Xiao L., A. Kirabo, J. Wu et al. 2015. Renal Denervation Prevents Immune Cell Activation and Renal Inflammation in Angiotensin II-Induced Hypertension. Circ. Res.117:547-57.

56. Banek C.T., M.M. Knuepfer, J.D.Foss et al. 2016. Resting Afferent Renal Nerve Discharge and Renal Inflammation: Elucidating the Role of Afferent and Efferent Renal Nerves in Deoxycorticosterone Acetate Salt Hypertension. Hypertension 68:1415-1423.

57. Zaldivia, M. T., J. Rivera, D. Hering et al. 2017. Renal Denervation Reduces Monocyte Activation and Monocyte-Platelet Aggregate Formation: An Anti-Inflammatory Effect Relevant for Cardiovascular Risk. Hypertension 69: 323-331.

58. Masuo K., H. Mikami, T. Ogihara \& M.L. Tuck. 1997. Sympathetic nerve hyperactivity precedes hyperinsulinemia and blood pressure elevation in a young, nonobese Japanese population. Am. J. Hypertens.10:77-83.

59. Grassi G., R. Dell'Oro, A. Facchini et al. 2004. Effect of central and peripheral body fat distribution on sympathetic and baroreflex function in obese normotensives. J. Hypertens. 22:2363-2369. 
60. Huggett R.J., E.M. Scott, S.G. Gilbey et al. 2003. Impact of type 2 diabetes mellitus on sympathetic neural mechanisms in hypertension. Circulation. 108:3097-3101.

61. Grassi G., R. Dell'Oro, F. Quarti-Trevano et al. 2005. Neuroadrenergic and reflex abnormalities in patients with metabolic syndrome. Diabetologia 48:1359-1365.

62. Scherrer U. \& C. Sartori.1997. Insulin as a vascular and sympathoexcitatory hormone: implications for blood pressure regulation, insulin sensitivity, and cardiovascular morbidity. Circulation. 96:4104-4113.

63. Bardgett M.E., J.J. McCarthy \& S.D. Stocker. 2010. Glutamatergic receptor activation in the rostral ventrolateral medulla mediates the sympathoexcitatory response to hyperinsulinemia. Hypertension. 55:284-290.

64. Lima N.K., F.Abbasi, C. Lamendola \& G.M. Reaven.2009. Prevalence of insulin resistance and related risk factors for cardiovascular disease in patients with essential hypertension. Am. J. Hypertens.22:106-111.

65. Esler M., N. Straznicky, N. Eikelis et al. 2006. Mechanisms of sympathetic activation in obesity-related hypertension. Hypertension 48:787-796.

66. Prichard B.N., B.A. Jager, J.H. Luszick et al.2002. Placebo-controlled comparison of the efficacy and tolerability of once-daily moxonidine and enalapril in mild to moderate essential hypertension. Blood Press. 11:166-172.

67. Grassi G., G. Seravalle, B.M. Cattaneo et al.1995. Sympathetic activation in obese normotensive subjects. Hypertension 25: 560-563.

68. Weyer C., R.E. Pratley, S. Snitker et al.2000. Ethnic Differences in Insulinemia and Sympathetic Tone as Links Between Obesity and Blood Pressure. Hypertension 36: 531537. 
69. Lambert E., N. Straznicky , T. Dawood et al.2011.Change in sympathetic nerve firing pattern associated with dietary weight loss in the metabolic syndrome. Front. Physiol. 2: 52.

70. Straznicky N., N. Eikelis, P. Nestel et al.2012. Baseline sympathetic nervous system activity predicts dietary weight loss in obese metabolic syndrome patients. J. Clin. Endocrinol. Metab. 97: 605-613.

71. Yakubu-Madus F.E., W.T. Johnson, K.M. Zimmerman et al.1999. Metabolic and hemodynamic effects of moxonidine in the Zucker diabetic fatty rat model of type 2 diabetes mellitus. Diabetes 48: 1093-1100.

72. Mahfoud F., M. Schlaich, I. Kindermann et al. 2011. Effect of renal sympathetic denervation on glucose metabolism in patients with resistant hypertension: a pilot study. Circulation 123: 1940-1946.

73. Kostinen H.A. \& Zierath J.R. 2002. Regulation of glucose transport in skeletal muscle. Ann. Med. 34: 410-418.

74. Jamerson K.A., S. Julius, T. Gudbrandsson, O. Anderson \& D.O. Brant. 1993. Reflex sympathetic activation induces acute insulin resistance in the human forearm. Hypertension 9: 618-623.

75. Julius S., M. Valentini \& P. Palatini. 2000. Overweight and Hypertension: A 2-Way Street? Hypertension 35: 807-813.

76. Thomopoulos C., H. Michalopoulou, A. Kasiakogias, A. Kefala \& T. Makris. 2011. Resistant hypertension and obstructive sleep apnea: the sparring partners. Int. J. Hypertens. 947246.

77. Rauscher H., D. Formanek, W. Popp \& H. Zwick.1993. Nasal CPAP and weight loss in hypertensive patients with obstructive sleep apnoea. Thorax 48: 529-533. 
78. Sharma S.K., S.Agrawal, D. Damodaran et al. 2011. CPAP for the metabolic syndrome in patients with obstructive sleep apnea. N. Engl. J. Med. 365: 2277-2286.

79. Witkowski A., A. Prejbisz, E. Florczak et al. 2011. Effects of renal sympathetic denervation on blood pressure, sleep apnea, and glycemic control in patients with resistant hypertension and sleep apnea. Hypertension 58: 559-565.

80. Worthley S.G., C.P. Tsioufis, M.I. Worthley et al. 2013. Safety and efficacy of a multielectrode renal sympathetic denervation system in resistant hypertension: the EnligHTN I trial. Eur. Heart. J. 34:2132-2140.

81. Davies J.E., C.H. Manisty, R. Petraco et al. 2013. First-in-man safety evaluation of renal denervation for chronic systolic heart failure: primary outcome from REACH-Pilot study. Int. J. Cardiol.162:189-192.

82. Pokushalov E., A. Romanov, G. Corbucci et al. 2012. A randomized comparison of pulmonary vein isolation with versus without concomitant renal artery denervation in patients with refractory symptomatic atrial fibrillation and resistant hypertension. J. Am. Coll. Cardiol. 60:1163-1170.

83. Brandt M.C., F. Mahfoud, S. Reda et al. 2012. Renal sympathetic denervation reduces left ventricular hypertrophy and improves cardiac function in patients with resistant hypertension. J. Am. Coll. Cardiol. 59:901-909.

84. Mahfoud F., B. Cremers, J. Janker et al. 2012. Renal hemodynamics and renal function after catheter-based renal sympathetic denervation in patients with resistant hypertension. Hypertension 60:419-424.

85. Schlaich M.P., N. Straznicky, M. Grima et al. 2011. Renal denervation: a potential new treatment modality for polycystic ovary syndrome? J. Hypertens. 29: 991-996. 
86. Yao Y., I.C. Fomison-Nurse, J.C. Harrison et al. 2014. Chronic bilateral renal denervation attenuates renal injury in a transgenic rat model of diabetic nephropathy. Am. J. Physiol. Renal. Physiol. 307: F251-F262.

87. Verloop W.L., W. Spiering, E.E. Vink et al. 2015. Denervation of the renal arteries in metabolic syndrome: the DREAMS-Study. Hypertension 65:751-757.

88. Brinkmann J., K. Heusser, B.M. Schmidt et al. 2012. Catheter-based renal nerve ablation and centrally generated sympathetic activity in difficult-to-control hypertensive patients: prospective case series. Hypertension 60:1485-1490.

89. Hering D., E.A. Lambert, P. Marusic et al. 2013. Substantial reduction in single sympathetic nerve firing after renal denervation in patients with resistant hypertension. Hypertension 61:457-464.

90. Henegar J.R., Y. Zhang, R. De Rama et al. 2014. Catheter-based radiofrequency renal denervation lowers blood pressure in obese hypertensive dogs. Am. J. Hypertens. 27:1285-1292.

91. Grassi G. 2013. Renal denervation in cardiometabolic disease: concepts, achievements and perspectives. Nutr. Metab. Cardiovasc. Dis. 23:77-83.

92. Asai K., G.P. Yang, Y.J. Geng et al. 1999. Beta-adrenergic receptor blockade arrests myocyte damage and preserves cardiac function in the transgenic $\mathrm{G}$ (alpha) mouse. J. Clin. Invest. 104:551-558.

93. Krum H., P. Sobotka, F. Mahfoud et al. 2011. Device-based antihypertensive therapy: therapeutic modulation of the autonomic nervous system. Circulation 123:209-215.

94. Esler MD, Böhm M, Sievert H, et al. 2014 Catheter-based renal denervation for treatment of patients with treatment-resistant hypertension: 36 month results from the SYMPLICITY HTN-2 randomized clinical trial. Eur Heart J. 35(26):1752-9. 
95. Mahfoud F, Böhm M, Schmieder R, et al. 2019 Effects of renal denervation on kidney function and long-term outcomes: 3-year follow-up from the Global SYMPLICITY Registry. Eur Heart J. pii: ehz118. doi: 10.1093/eurheartj/ehz118

96. Sardar P, Bhatt DL, Kirtane AJ, et al. 2019 Sham-Controlled Randomized Trials of Catheter-Based Renal Denervation in Patients With Hypertension. J Am Coll Cardiol. 73(13):1633-1642

97. Wallbach M, Lehnig LY, Helms HJet al. 2015 Long term effects of baroreflex activation therapy on glucose metabolism. Acta Diabetol. 52(5):829-35

98. Lohmeier TE TE $^{1}$ Dwyer TM, Irwin ED, et al. 2007 Prolonged activation of the baroreflex abolishes obesity-induced hypertension. Hypertension. 49(6):1307-14

\section{Figure Legends:}


Figure 1: Sympathetic overdrive induces immune activation that causes inflammation and organ damage. The immune cascade is sustained by the bidirectional interaction between the sympathetic nervous system and immune mechanisms causing immune infiltration and immune-mediated inflammatory damage in the organs regulating blood pressure to result in the derangement of cardiovascular and metabolic diseases.

Figure 2: Comparison of 24-hr systolic and diastolic blood pressure (SBP and DBP) changes in renal denervation vs sham-control groups in the 3 recent randomized, sham-controlled clinical trials showed. ABPM indicates ambulatory BP monitoring; CI, confidence interval; HTN-ON MED, Spyral Hypertension on Medication trial; HTN-OFF MED, Spyral Hypertension OFF Medication trial; and RADIANCE-HTN SOLO, RADIANCE hypertension solo (off medication) trial. Reprinted from Kandzari et al, 1 Azizi et al, 2 and Townsend et al 3 with permission. Copyright (C) 2018, Elsevier.

Figure 3: Renal Sympathetic Innervation includes efferent sympathetic fibres originating in the central nervous system and traversing to the kidney as well as afferent sensory fibres from the kidneys to hypothalamus. Increased sympathetic outflow to the renal vascular bed reduces renal blood flow, releases renin, thereby activating the renin-angiotensin-aldosterone (RAAS) cascade and enhances tubular reabsorption of urinary sodium and water. The cardiometabolic consequence of increased sympathetic flow includes cardiac hypertrophy and arrhythmias, endothelial dysfunction and insulin resistance in the skeletal muscle contributing to the development of diabetes mellitus and metabolic syndrome. 